\title{
HMGR overexpression and interference affects the expression of steroidogenic genes and cholesterol content in bovine intramuscular adipocytes
}

\section{Xiaomu Liu}

Shandong Academy of Agricultural Sciences

\section{Wei You}

Shandong Academy of Agricultural Sciences

\section{Xianglun Zhang}

Shandong Academy of Agricultural Sciences

\section{Qing Jin}

Shandong Academy of Agricultural Sciences

\section{Xiuwen Tan}

Shandong Academy of Agricultural Sciences

Chen Wei

Shandong Academy of Agricultural Sciences

Hongbo Zhao

Shandong Academy of Agricultural Sciences

Chen Zhang

Shandong Academy of Agricultural Sciences

Yifan Liu

Shandong Academy of Agricultural Sciences

\section{Fachun Wan}

Shandong Academy of Agricultural Sciences

guifen liu ( $\square$ liuguifen126@126.com )

Shandong Academy of Agricultural Sciences https://orcid.org/0000-0003-3300-8028

\section{Research article}

Keywords: HMGR, Overexpression, Interference, Cholesterol, Bovine

Posted Date: June 12th, 2019

DOl: https://doi.org/10.21203/rs.2.10141/v1 
License: (c) (i) This work is licensed under a Creative Commons Attribution 4.0 International License. Read Full License 


\section{Abstract}

Background:Previously, we found that mevalonic acid stimulates HMGR expression in bovine intramuscular adipocytes, and influences adipocyte differentiation. However, it remains unclear whether there is any direct link between HMGR, steroidogenic genes, and cholesterol content. RNA-Seq was conducted to determine the differences between the gene expression profiles of bovine adipocytes containing different HMGR expression constructs. Results:In total, 10234 differentially expressed genes (DEGs) were found. Of these, 35 and 6 DEGs between the control and the overexpression groups were functionally related to lipid and energy metabolisms, respectively. Additionally, 43 and 8 DEGs between the control and the HMGR inhibition groups were related to lipid and energy metabolism. Additionally, several DEGs related to lipid and energy metabolism were identified between the HMGR overexpression group and the HMGR interference group. Several DEGs correlated positively or negatively with overexpression or inhibition of HMGR. We also found that, following activation or inhibition of the HMGR gene, AMPK and SIRT1 had opposite expression patterns in bovine intramuscular adipocytes. Interestingly, the HMGR gene was downregulated when HMGR was overexpressed, and upregulated when HMGR was inhibited. Conclusion:Our findings establish a theoretical understanding of signaling pathways involved in cholesterol synthesis by elucidating the relationships between key genes.

\section{Background}

Intramuscular fat (IMF) is an important factor influencing meat quality in beef cattle [1-4]. The right amount of IMF can enhance meat quality traits such as flavor, juiciness, and tenderness $[5,6]$. One of the important traits in beef that researchers have focused on are the mechanisms and regulation of fat deposition in fat tissue following increased or decreased fat intake, which may be related to concentrations of circulating cholesterol $[7,8]$. With increasing concerns regarding the relationship between fat intake and health, consumers have become more conscious of what constitutes a healthy diet, and are increasingly demanding products with reduced cholesterol content [ 9]. Due to the high cholesterol content and the appealing taste of IMF, beef cattle are considered to be an attractive candidate for cholesterol reduction and dietary improvement.

Three-hydroxy-3-methyl-glutaryl-coenzyme A reductase (HMGR), is a rate-limiting enzyme of cholesterol synthesis. This protein can catalyze the conversion of 3-hydroxy-3-methyl-glutaryl-coenzyme A (HMGCoA) to mevalonate, and is a key factor in the regulation of cholesterol content in vivo [ 10,11$]$. Sirtuin type 1 (SIRTI), AMP-activated protein kinase (AMPK), and lipoprotein lipase (LPL) play important roles in the regulation of the energy metabolism. Previous studies have shown that long-term moderate caloric restriction and resveratrol supplementation can improve lipid disorders and decrease fat accumulation, by regulating the SIRT1-autophagy pathway [12]. However, it remains unclear whether there is any direct link between HMGR, steroidogenic genes, and cholesterol content.

Cholesterol content is a complex genetic trait. The genetics underlying the biochemical process and molecular background that determine cholesterol content are not yet fully understood, particularly with 
regard to local Chinese cattle breeds. Using RNA-Seq analysis to select differently expressed genes (DEGs) correlated with cholesterol levels, the present study aimed to explore the effects of HMGR overexpression and interference on the expression patterns of cholesterol-related genes, as well as on differential gene expression in bovine intramuscular adipose cells.

\section{Materials And Methods}

\section{Cell culture and induction of preadipocyte differentiation}

Cells from intramuscular fat tissue of Lilu cattle were isolated according to the method described by Liu et al. [13]. Cattles were raised in the Shandong Provincial Testing Center of Beef Cattle Performance (Shandong Province, Jinan, China). The cells were cultured and maintained in DMEM/F12 (HyClone, UT, USA) containing $10 \%$ fetal bovine serum (FBS; Gibco, NY, USA), an antibiotic-antimycotic agent (100 U/ml penicillin and $100 \mu \mathrm{g} / \mathrm{ml}$ streptomycin; Gibco). Cells were seeded at $2 \times 10^{4} \mathrm{cells} / \mathrm{cm}^{2}$ and incubated at $37^{\circ} \mathrm{C}$ in a humidified $5 \% \mathrm{CO}_{2}$ environment. To induce adipogenesis, two-day post-confluent preadipocytes (designated as day zero) were incubated for $72 \mathrm{~h}$ in differentiation-inducing medium with DMEM/F12, 4 $\mu \mathrm{g} / \mathrm{ml}$ insulin (Sigma, St. Louis, MO, USA), $0.1 \mathrm{mg} / \mathrm{ml}$ dexamethasone (DEX; Sigma), and $0.1 \mathrm{mg} / \mathrm{ml}$ 3isobutyl-1-methylxanthine (IBMX; Sigma). The cells were then transferred into DMEM/F12 containing 1 $\mathrm{mM}$ octanoate (Sigma), $10 \mathrm{mM}$ acetate (Sigma), $10 \mu \mathrm{g} / \mathrm{ml}$ transferrin (Sigma), $3 \mu \mathrm{g} / \mathrm{ml}$ cholesterol (Sigma), $17 \mathrm{mM}$ biotin (Sigma), $100 \mathrm{mM}$ calcium pantothenate (Sigma), and 0.5\% bovine serum albumin (BSA). After induction, the experimental cell groups were defined as follows: 1 ) adipogenic group (control group), 2) adipogenic + overexpression negative control (NC) group (viral transduction control group), 3) adipogenic + HMGR overexpression group, 4) adipogenic + interference NC group (transfected with an unrelated sequence), and 5) adipogenic + HMGR interference group

The animals used in this study were reared and sacrificed in compliance with national regulations for the humane care and use of animals in research (China Administration Rule of Laboratory Animals, Operating Procedure of Cattle Slaughtering GB/T 19477-2004).

\section{Oil Red 0 staining}

To examine lipid accumulation, cells were seeded in six-well culture plates at $5 \times 10^{4} / \mathrm{cm}^{2}$. At eight days of incubation, the medium was removed, and the cells were washed three times with phosphate-buffered saline (PBS; Beijing Dingguo Changsheng Biotechnology Co. Ltd. Beijing, China) and fixed with 10\% formaldehyde for $30 \mathrm{~min}$ at room temperature. After washing with PBS, the cells were stained for at least $1 \mathrm{~h}$ with $1 \%$ filtered Oil Red O (6:4 ratio of Oil Red O stock solution to $\mathrm{H}_{2} \mathrm{O}$, whereby the Oil Red $\mathrm{O}$ stock solution comprised 0.5\% Oil Red O in isopropyl alcohol). Oil Red O was obtained from Beijing Dingguo Changsheng Biotechnology Co. Ltd. Morphological features of cells were examined by microscopy (Olympus Corporation, Japan). 


\section{Construction of the HMGR expression vector}

To determine the effects of HMGR on cholesterol synthesis, bovine intramuscular adipocytes were transfected with recombinant adenoviral vectors (Beijing Dingguo Changsheng Biotechnology Co. Ltd.). The recombinant vector ADV4-HMGR was then transfected into adipose cells via the method described by Hofgen and Willmitzer [ 14].

\section{siRNA transfection}

The sequence of the small interfering RNA (siRNA) for HMGR inhibition was as follows $\left(5^{\prime}-3^{\prime}\right)$ : sense, GUUCUAACUCACAGGAUGATT; and antisense, UCAUCCUGUGAGUUAGAACTT. The siRNA oligonucleotides were designed by Beijing Dingguo Changsheng Biotechnology Co. Ltd. The siRNA inhibition group and siRNA negative control group were transfected for $48 \mathrm{~h}$ using the lipofectamine transfection reagent (11668-500; Invitrogen), according to the manufacturer's protocol.

\section{Detection of DEGs}

The generation and processing of raw RNA-seq data was conducted by the Beijing Genomics Institute (Beijing, China) using the lllumina HiSeq platform. We detected DEGs using the PossionDis algorithm, which is based on the Poisson distribution, and was performed as described by Audic et al. [15]. The parameters to determine significant changes in gene were as follows: fold change $\geq 2$ and a false discovery rate $(\mathrm{FDR}) \leq 0.001$.

\section{Pathway analysis of DEGs}

DEGs were annotated according to the Kyoto Encyclopedia of Genes and Genomes (KEGG) database. We performed pathway enrichment analysis using phyper, a function in R based on hypergeometric distribution (https://cran.r-project.org/). We then calculated the FDR for each p-value, and considered an FDR $<0.001$ to represent significant enrichment.

\section{Quantification of total cholesterol in adipose cells}

Total cholesterol (TC), high-density lipoprotein cholesterol (HDL-C), and low-density lipoprotein cholesterol (LDL-C) levels were measured in adipose cells after seeding in a six-well culture plate, using detection kits specific to each cholesterol type (T-CHO kit, HDL-C kit, and LDL-C kit; Nanjing Jiancheng Biology Institute, Nanjing, China).

\section{Total RNA isolation and quantitative real-time PCR}


Total RNA was isolated form adipose cells using the TRIzol ${ }^{\circledR}$ reagent (15596-018; Invitrogen, CA, USA), and then converted into cDNA using the PrimeScript ${ }^{\circledR}$ RT Reagent Kit (DRR027A; TaKaRa, Shanghai, China). Expression of selected genes was then analyzed using the Fast SYBR® Green Master Mix Bulk Pack (4385614; Invitrogen, USA). The following primers were used for quantitative real-time PCR (qRTPCR): HMGR sense, 5'-TGCTGGTGCTGAGTATGTGG-3' and antisense, 5'-CATTCTACAAGAGCATCCAGG-3';

LPL sense, 5'-GACACTTGCCACCTCATTC-3' and antisense, 5'-CATCCGCCATCCAGTTCATA-3'; AMPK sense, 5'-CCGTATTATTTGCGTGTTCG-3' and antisense, 5'-TGTGGCGTAGCAGTCCCT-3'; SIRT1 sense, 5'TGCAATAGACTTTCCAGACC-3' and antisense, 5'-GTGTATCTATGTTCTGAGT-3'; and GAPDH sense, 5'TGCTGGTGCTGAGTATGTGG-3' and antisense, 5'-GATGATGACCCTCTTGGCG-3' (Beijing Dingguo Changsheng Biotechnology Co. Ltd.). Three replicates were used for each experiment. Relative mRNA expression levels were measured using the comparative Ct method $(\Delta \Delta \mathrm{Ct})$, with GAPDH as the internal control.

\section{Western blotting}

Cell extracts were isolated in RIPA buffer $\left(1 \mathrm{ml}\right.$ per $10^{7}$ cells $/ 100 \mathrm{~mm}$-dish/150 $\mathrm{cm}^{2}$-flask; $0.5 \mathrm{ml}$ per $5 \times$ $10^{6}$ cells/60 mm-dish/75 cm²-flask; WB-0071; Beijing Dingguo Changsheng Biotechnology Co. Ltd.). The Pierce BCA Assay (BCA01; Beijing Dingguo Changsheng Biotechnology Co. Ltd.) was used to determine protein concentration. Samples $(50-100 \mu \mathrm{g})$ were separated using $10 \%$ sodium dodecyl sulfate polyvinylidene fluoride (SDS-PVDF) gels, and then transferred to PVDF membranes (XLL092-2; Pall, USA). The membranes were incubated with anti- $\beta$-actin (SC-47778; Santa Cruz Biotechnology, Inc., Santa Cruz, CA, USA), anti-HMGR (Ab174830; Abcam, Cambridge, United Kingdom), anti-AMPK (Ab3759; Abcam), anti-LPL (Ab21356; Abcam), and anti-SIRT1 (Bs-0921R; Bioss Biotechnology, Inc., Beijing, China) antibodies in blocking buffer for $2 \mathrm{~h}$ at room temperature. After the membranes were incubated with secondary antibodies (Santa Cruz Biotechnology, Inc.), the enhanced chemiluminescence system was used to detect immunoreactive bands.

\section{Statistical analysis}

Differences in gene expression between the experimental groups were calculated using an analysis of variance (ANOVA), followed by a Bonferroni test. Results were considered statistically significant when $P$ $<0.05$.

\section{Results}

\section{Adipose cell morphology and lipid accumulation}

We first evaluated morphological changes in induced adipogenic bovine intramuscular adipocytes, and stained cells with Oil Red 0 to visualize lipid droplet accumulation after induction. As shown in Figure 1 , 
induced adipocytes were large, round, and filled with fat droplets, compared to immature adipocytes.

\section{DEG detection}

We sequenced RNA from the five experimental groups using the Illumina HiSeq platform, and generated an average of $6.58 \mathrm{~Gb}$ for each sample. As showed in Figure 2, after mapping sequenced reads to the reference genome and reconstructing the transcripts, we obtained 13264 novel transcripts from all samples. Of these, 9444 were previously unknown splicing events for known genes, 862 were novel coding transcripts without any known features, and the remaining 2958 were long noncoding RNAs.

We then analyzed differential gene expression between samples. The summary of DEGs is shown in Figure 2. There were a total of $10234 \mathrm{DEGs}$, and this number was higher when the HMGR gene was overexpressed or suppressed. There were 1554 DEGs found in the HMGR overexpression group vs. the HMGR interference group, where genes were mostly upregulated. We also found that there were more upregulated genes within DEGs between all experimental groups.

\section{Pathway analysis of DEGs}

We performed KEGG pathway classification and functional enrichment analyses for DEGs (Figure 3). These DEGs were found to be mostly involved in signal transduction, cancers, infectious disease, global and overview maps, and the immune system. As shown in Figure 3a and 3b, 35 DEGs after HMGR overexpression, and 43 DEGs after HMGR inhibition, were functionally involved in lipid metabolism. Furthermore, 6 DEGs after HMGR overexpression, and 8 DEGs after HMGR inhibition, were functionally involved in energy metabolism.

\section{AMPK signaling}

Changes in gene expression relevant to the AMPK signaling pathway following over-expression of the HMGR gene are shown in Figure 4a and 4b. Interestingly, the HMGR gene was downregulated when HMGR was overexpressed, whereas the SIRT1 gene was upregulated. In contrast, the HMGR gene itself was upregulated following HMGR interference.

\section{TC, LDL-C, and HDL-C content in adipose cells}

To understand the impact of HMGR overexpression on the amount of TC, LDL-C, and HDL-C, we examined the levels of these types of cholesterol in the five experimental groups. In contrast with other groups, the HMGR overexpression group had the highest amounts of TC, LDL-C, and HDL-C, particularly for TC and HDL-C (Figure 5). 


\section{qRT-PCR of HMGR, AMPK, SIRT1, and LPL}

To investigate the relationship between HMGR expression and cholesterol synthesis in bovine adipose cells, expression levels of HMGR, AMPK, SIRT1, and LPL, which are four steroidogenesis-related genes, were assessed in adipose tissue using qRT-PCR (Figure 6).

For HMGR, the overexpression group showed higher mRNA expression levels than those in other groups, with the HMGR interference group showing the lowest levels out of all groups. AMPK mRNA expression was lowest when HMGR was overexpressed; even though AMPK protein expression levels were increased (as shown in Figure 7). SIRT1 showed similar results as AMPK, whereas LPL expression levels were higher when HMGR was overexpressed, and lower when HMGR was inhibited.

\section{Western blotting of HMGR, AMPK, SIRT1, and LPL}

To investigate changes in protein expression, we performed western blotting in the five experimental groups (Figure 7). The results showed that HMGR protein expression in the HMGR overexpression group was higher than that in the other four groups. AMPK protein expression levels showed a little differences between the groups. SIRT-1 and LPL protein expression levels were higher in the HMGR interference group, and lower in the HMGR overexpression group.

\section{Discussion}

Adipose tissue is the major depot for energy and cholesterol storage, with most of the intracellular cholesterol distributed in the form of lipid droplets $[16,17]$. Cholesterol homeostasis may have a role in the regulation of adipocyte size and function $[18,19]$. In addition, cellular cholesterol homeostasis is involved in various diseases of nongenetic origin, such as atherosclerosis, leading to lipid accumulation in target organs [ 18,20$]$.

In the present study, DEG and KEGG pathway analyses were performed to explore the regulatory network underlying bovine adipose cell following HMGR overexpression and interference. As expected, several well-known pathways [21] related to lipid metabolism were identified, particularly the AMPK signaling pathway. In AMPK signaling, the HMGR, AMPK, and SITR1 genes play a functional role in lipid metabolism or cholesterol biosynthesis.

In terms of cholesterol levels, we found that those of TC, LDL-C, and HDL-C were highest when the HMGR gene was overexpressed; however, their levels were not majorly decreased when HMGR was inhibited. When we investigated protein expression of HMGR, AMPK, SIRT-1, and LPL, we found that HMGR protein levels were higher in the HMGR overexpression group compared to those in the control group. However, SIRT-1 protein levels were lower in the HMGR overexpression group compared to those in the HMGR interference group. Therefore, the results obtained for protein expression were consistent with those obtained for gene expression. 
Given that HMGR is a rate-limiting enzyme in cholesterol biosynthesis [10], we generated an adipocyte gene expression dataset using RNA-Seq, and examined the relationship between steroidogenic genes and cholesterol levels by overexpressing or inhibiting the HMGR gene. Transcriptional and pathway analysis results showed that overexpression of HMGR correlated with downregulation of AMPK and SIRT1 gene expression.

AMPK is a crucial energy sensor that maintains energy homeostasis. It is also a major regulator of the lipid metabolism through phosphorylation and inactivation of numerous metabolic enzymes, including HMGR [ 22, 23]. In adipose tissue, the chronic activation of AMPK led to downregulation of HMGR expression in a new transgenic mouse model [24]. The 5-aminoimidazole-4-carboxamide (AICA) ribonucleotide was shown to induce activation of AMPK, and directly inhibit the expression of HMGR, one of its target genes [22]. In the present study, HMGR mRNA expression was reduced when it was overexpressed in bovine adipose cells. In contrast, HMGR expression was increased when the HMGR gene was inhibited. These results are important, given the key role of the HMGR gene in the process of cholesterol synthesis.

SIRT1 is a longevity-associated deacetylase enzyme that modulates metabolic homeostasis in response to cellular energy. AMPK and SIRT1 are related proteins and share common target pathways [25]. The present study showed that the expression of SIRT1 decreased when the HMGR gene was overexpressed. However, SIRT1 was upregulated when HMGR expression was modified. These results imply that the HMGR and SIRT1 genes have contrasting roles in the cholesterol synthesis pathway.

The LPL gene encodes a rate-limiting enzyme that has a key role in the hydrolysis of triglycerides. LPL deficiency and dysfunction is associated with many disorders of the lipoprotein metabolism [26, 27]. In the present study, our results showed that the patterns of LPL gene expression were consistent with those of HMGR expression.

\section{Conclusions}

This study showed that both overexpression and interference of HMGR in bovine intramuscular adipocytes could regulate the expression levels of relevant genes. Several DEGs correlated positively or negatively with HMGR overexpression or inhibition. Furthermore, AMPK and SIRT1 showed opposite expression patterns in bovine intramuscular adipocytes following overexpression or inhibition of HMGR. These findings shed light on the signaling pathways involved in the cholesterol synthesis process, and elucidate how cholesterol levels may be controlled for future beef commercial production via a multi-gene pyramiding system.

\section{Declarations}

\section{Abbreviations}


Not applicable.

\section{Ethics approval and consent to participate}

Not applicable.

\section{Consent for publication}

Not applicable.

\section{Availability of data and material}

Not applicable.

\section{Competing interests}

The authors declare that they have no competing interest.

\section{Funding}

This work was supported by Young Talents Training Program of Shandong Academy of Agricultural Science, Agricultural Science and Technology Innovation Project of Shandong Academy of Agricultural Sciences (CXGC2018E10), the National Natural Science Foundation of China (grants no. 31601966 and no. 31100890), and CARS-37.

\section{Authors' contributions}

Guifen Liu and Fachun Wan designed the experiments. Xiaomu Liu and Wei You drafted the manuscript. Xianglun Zhang, Qing Jin, Xiuwen Tan, Chen Wei, Hongbo Zhao, Chen Zhang and Yifan Liu performed the experiments.

\section{Acknowledgements}

Not Applicable.

\section{References}

1. Cui H, Liu XRR, Zhao GP, Zheng MQ, Chen JL, Wen J (2012) Identification of 
differentially expressed genes and pathways for intramuscular fat deposition in pectoralis major tissues of fast-and slow-growing chickens. BMC genomics 13:213

2. Fernandez X, Monin G, Talmant A, Mourot J, Lebret B (1999) Influence of intramuscular fat content on the quality of pig meat-1. Composition of the lipid fraction and sensory characteristics of $\mathrm{m}$. longissimus lumborum. Meat science 53(1):59-65

3. Fernandez X, Monin G, Talmant A, Mourot J, Lebret B (1999) Influence of intramuscular fat content on the quality of pig meat - 2. Consumer acceptability of m. longissimus lumborum. Meat science 53(1):67-72

4. Rincker PJ, Killefer J, Ellis M, Brewer MS, McKeith FK (2008) Intramuscular fat content has little influence on the eating quality of fresh pork loin chops. Journal of animal science 86(3):730-737

5. Soret B, Mendizabal JA, Arana A, Alfonso L (2016) Expression of genes involved in adipogenesis and lipid metabolism in subcutaneous adipose tissue and longissimus muscle in low-marbled Pirenaica beef cattle. Animal : an international journal of animal bioscience 10(12):2018-2026

6. Hocquette JF, Gondret F, Baeza E, Medale F, Jurie C, Pethick DW (2010) Intramuscular fat content in meat-producing animals: development, genetic and nutritional control, and identification of putative markers. Animal : an international journal of animal bioscience $4(2): 303-319$

7. da Costa AS, Pires VM, Fontes CM, Mestre Prates JA (2013) Expression of genes controlling fat deposition in two genetically diverse beef cattle breeds fed high or low silage diets. BMC veterinary research 9:118

8. Haberka M, Okopien B, Gasior Z (2016) Obesity, ultrasound indexes of fat depots and lipid goal attainment in patients with high and very high cardiovascular risk: A novel approach towards better risk reduction. Nutrition, metabolism, and cardiovascular diseases : NMCD 
26(2):123-133

9. Ospina EJ, Sierra CA, Ochoa O, Perez-Alvarez JA, Fernandez-Lopez J (2012)

Substitution of saturated fat in processed meat products: a review. Critical reviews in food science and nutrition 52(2):113-122

10. Istvan ES, Deisenhofer J (2000) The structure of the catalytic portion of human HMG-CoA reductase. Biochimica et biophysica acta 1529(1-3):9-18

11. Jiang J, Kai G, Cao X, Chen F, He D, Liu Q (2006) Molecular cloning of a HMG-CoA reductase gene from Eucommia ulmoides Oliver. Bioscience reports 26(2):171-181

12. Ding S, Jiang J, Zhang G, Bu Y, Zhang G, Zhao X (2017) Resveratrol and caloric restriction prevent hepatic steatosis by regulating SIRT1-autophagy pathway and alleviating endoplasmic reticulum stress in high-fat diet-fed rats. PloS one 12(8):e0183541

13. Liu X, You W, Cheng H, Zhang Q, Song E, Wan F, Han H, Liu G (2016) Effect of mevalonic acid on cholesterol synthesis in bovine intramuscular and subcutaneous adipocytes. Journal of applied genetics 57(1):113-118

14. Hofgen R, Willmitzer L (1988) Storage of competent cells for Agrobacterium transformation. Nucleic acids research 16(20):9877

15. Audic S, Claverie JM (1997) The significance of digital gene expression profiles. Genome research 7(10):986-995

16. Farkas J, Angel A, Avigan MI (1973) Studies on the compartmentation of lipid in adipose cells. II. Cholesterol accumulation and distribution in adipose tissue components. Journal of lipid research 14(3):344-356

17. Zhu Y, Chen CY, Li J, Cheng JX, Jang M, Kim KH (2018) In vitro exploration of ACAT contributions to lipid droplet formation during adipogenesis. Journal of lipid research 59(5):820-829 
18. Le Lay S, Krief S, Farnier C, Lefrere I, Le Liepvre X, Bazin R, Ferre P, Dugail I

(2001) Cholesterol, a cell size-dependent signal that regulates glucose metabolism and gene expression in adipocytes. The Journal of biological chemistry 276(20):16904-16910

19. Shao F, Wang X, Yu J, Jiang H, Zhu B, Gu Z (2014) Expression of miR-33 from an

SREBF2 intron targets the FTO gene in the chicken. PloS one 9(3):e91236

20. Khera AV, Demler OV, Adelman SJ, Collins HL, Glynn RJ, Ridker PM, Rader DJ,

Mora S (2017) Cholesterol Efflux Capacity, High-Density Lipoprotein Particle Number, and Incident Cardiovascular Events: An Analysis From the JUPITER Trial (Justification for the Use of Statins in Prevention: An Intervention Trial Evaluating Rosuvastatin). Circulation 135(25):2494-2504

21. Kokta TA, Dodson MV, Gertler A, Hill RA (2004) Intercellular signaling between adipose tissue and muscle tissue. Domestic animal endocrinology 27(4):303-331 22. Liu S, Jing F, Yu C, Gao L, Qin Y, Zhao J (2015) AICAR-Induced Activation of AMPK Inhibits TSH/SREBP-2/HMGCR Pathway in Liver. PloS one 10(5):e0124951 23. Hardie DG, Ross FA, Hawley SA (2012) AMPK: a nutrient and energy sensor that maintains energy homeostasis. Nature reviews Molecular cell biology 13(4):251-262 24. Knowles C, Liu ZM, Yang J (2011) Compensatory increase in lipogenic gene expression in adipose tissue of transgenic mice expressing constitutively active AMP-activated protein kinase-alpha1 in liver. Biochemical and biophysical research communications 412(2):249-252 25. Arab Sadeghabadi Z, Nourbakhsh M, Pasalar P, Emamgholipour S, Golestani A, Larijani B, Razzaghy-Azar M (2018) Reduced gene expression of sirtuins and active AMPK levels in children and adolescents with obesity and insulin resistance. Obesity research \& clinical practice 12(2):167-173

26. Xie C, Wang ZC, Liu XF, Yang MS (2010) The common biological basis for 
common complex diseases: evidence from lipoprotein lipase gene. European journal of human genetics : EJHG 18(1):3-7

27. Socquard E, Durlach A, Clavel C, Nazeyrollas P, Durlach V (2006) Association of HindIII and Pvull genetic polymorphisms of lipoprotein lipase with lipid metabolism and macrovascular events in type 2 diabetic patients. Diabetes \& metabolism 32(3):262-269

\section{Figures}
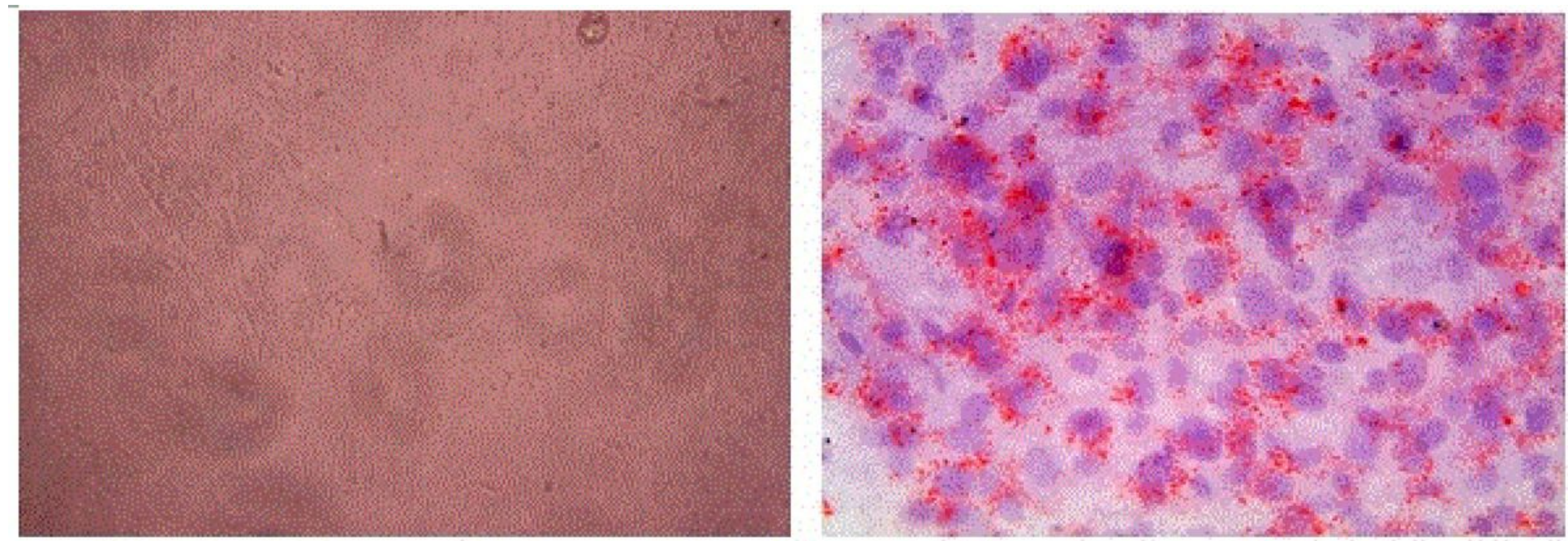

Figure 1

Oil Red 0 staining of bovine adipose cells. Original magnification: $\times 400$. 


\section{0}

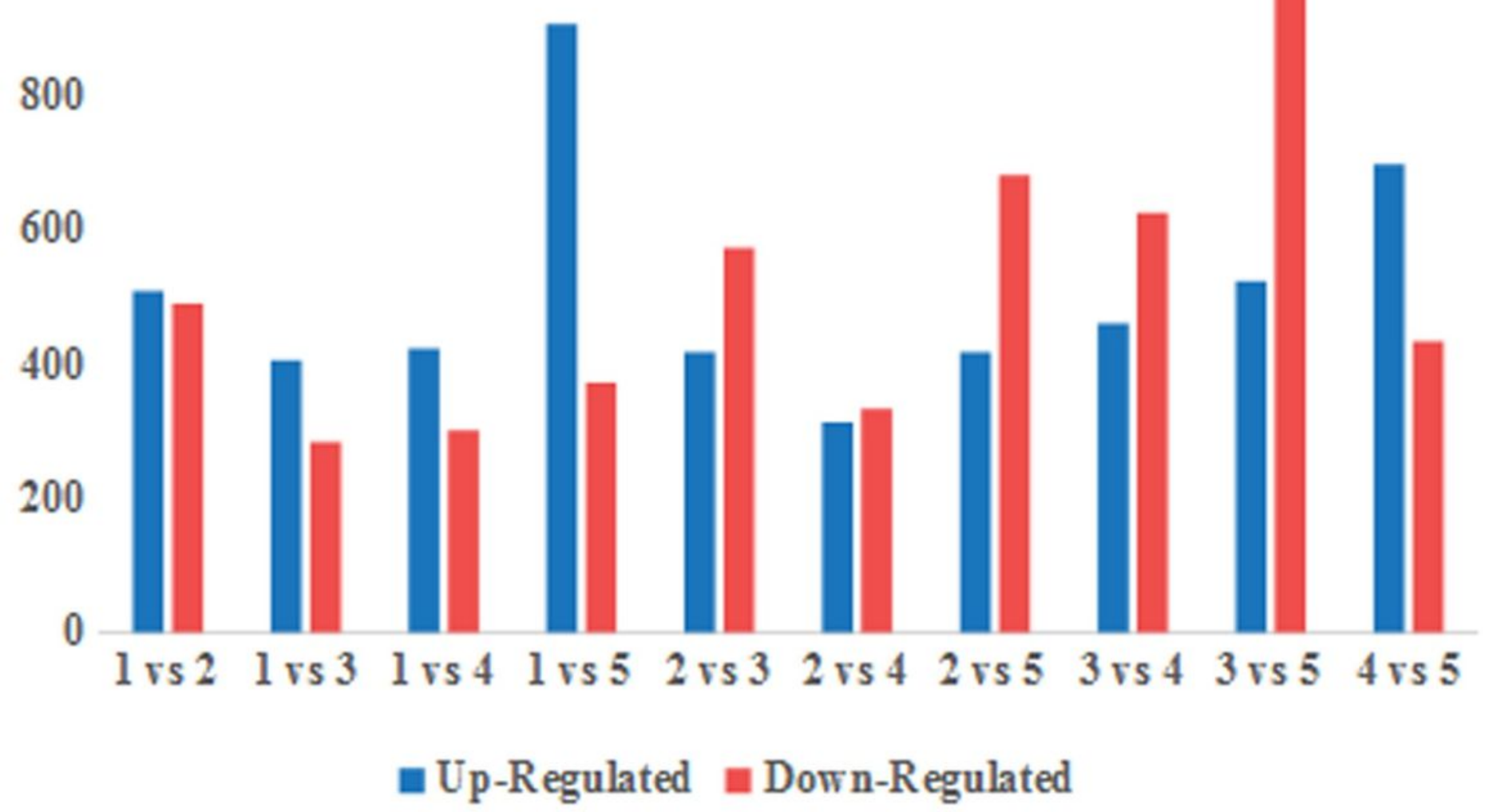

Figure 2

Summary of DEGs. The $X$ axis shows the compared samples. The $Y$ axis shows DEG numbers. The red color represents upregulated DEGs, and the blue color represents downregulated DEGs. 1: adipogenic group (control group), 2: adipogenic + overexpression NC, 3: adipogenic + HMGR overexpression group, 4: adipogenic + interference NC, and 5: adipogenic + HMGR interference group. DEGs, differentially expressed genes. 

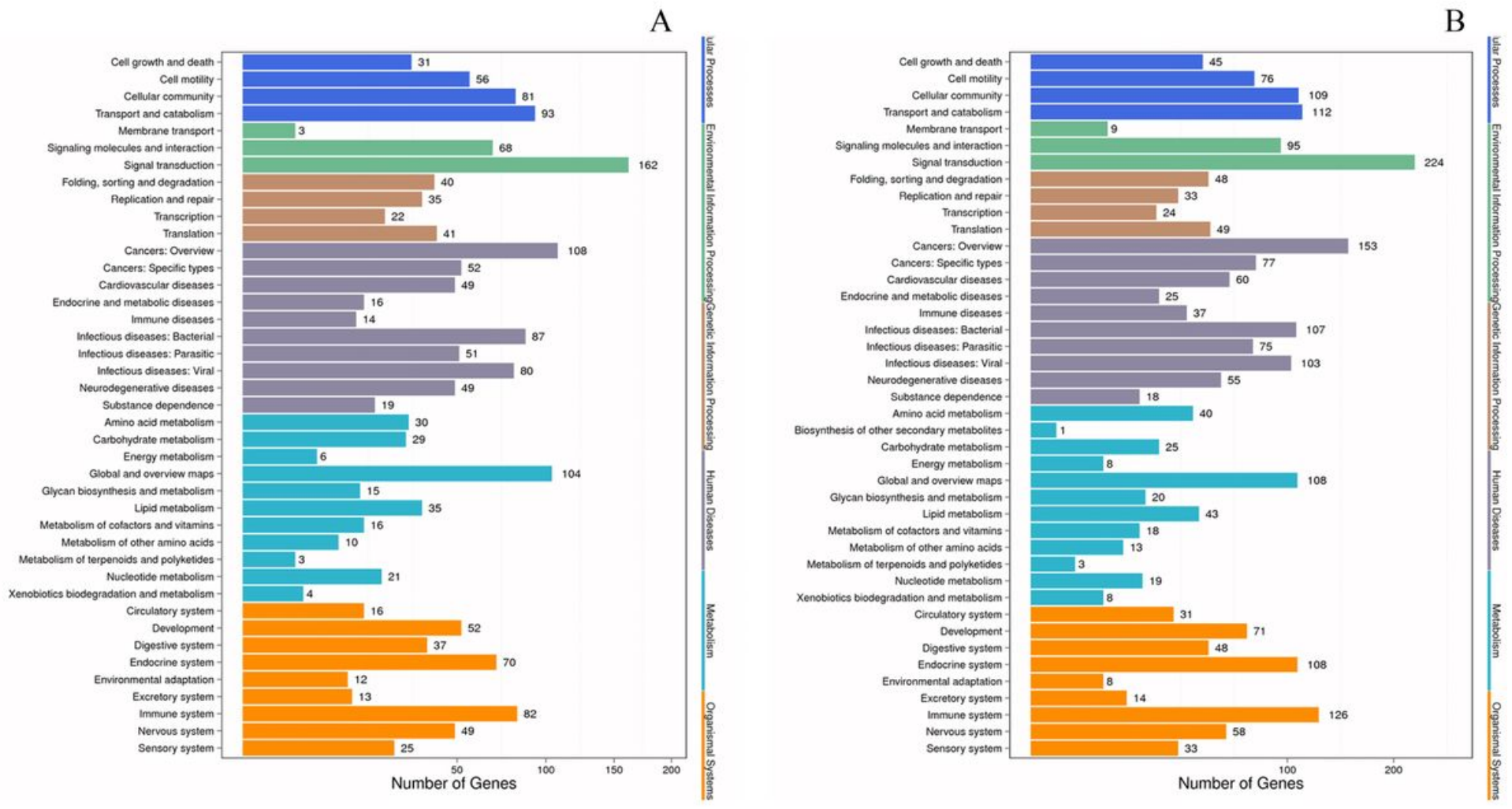

Figure 3

Pathway annotation of DEGs. The $X$ axis shows the number of DEGs. The $Y$ axis shows the pathway names. Left column: adipogenic group (control group) vs. adipogenic + HMGR overexpression group. Right column: adipogenic group (control group) vs. adipogenic + HMGR interference group. DEGs, differentially expressed genes. 


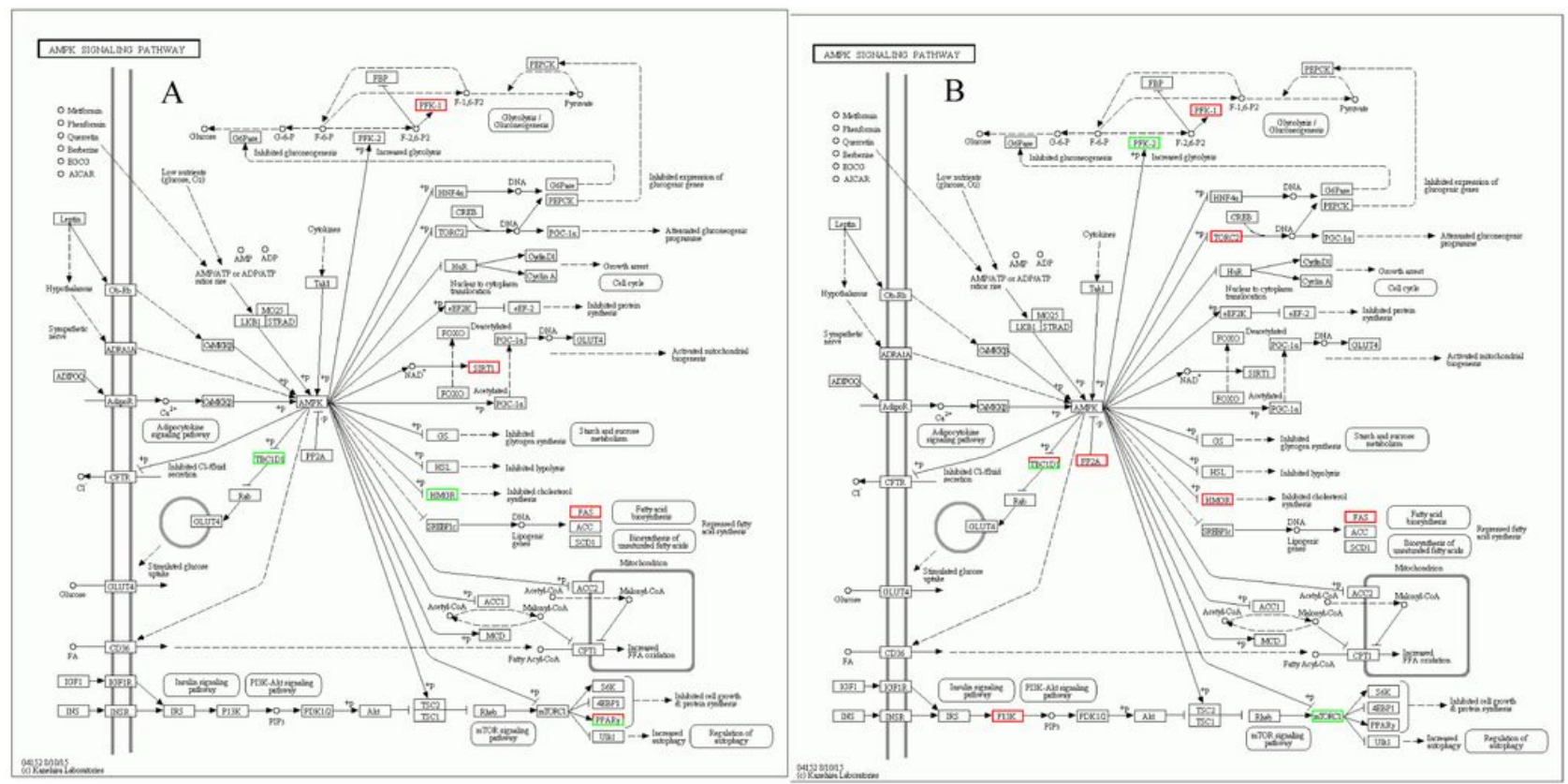

Figure 4

The AMPK signaling pathway. Upregulated genes are marked with red borders and downregulated genes with green borders. Non-differentially expressed genes are marked with black borders. 


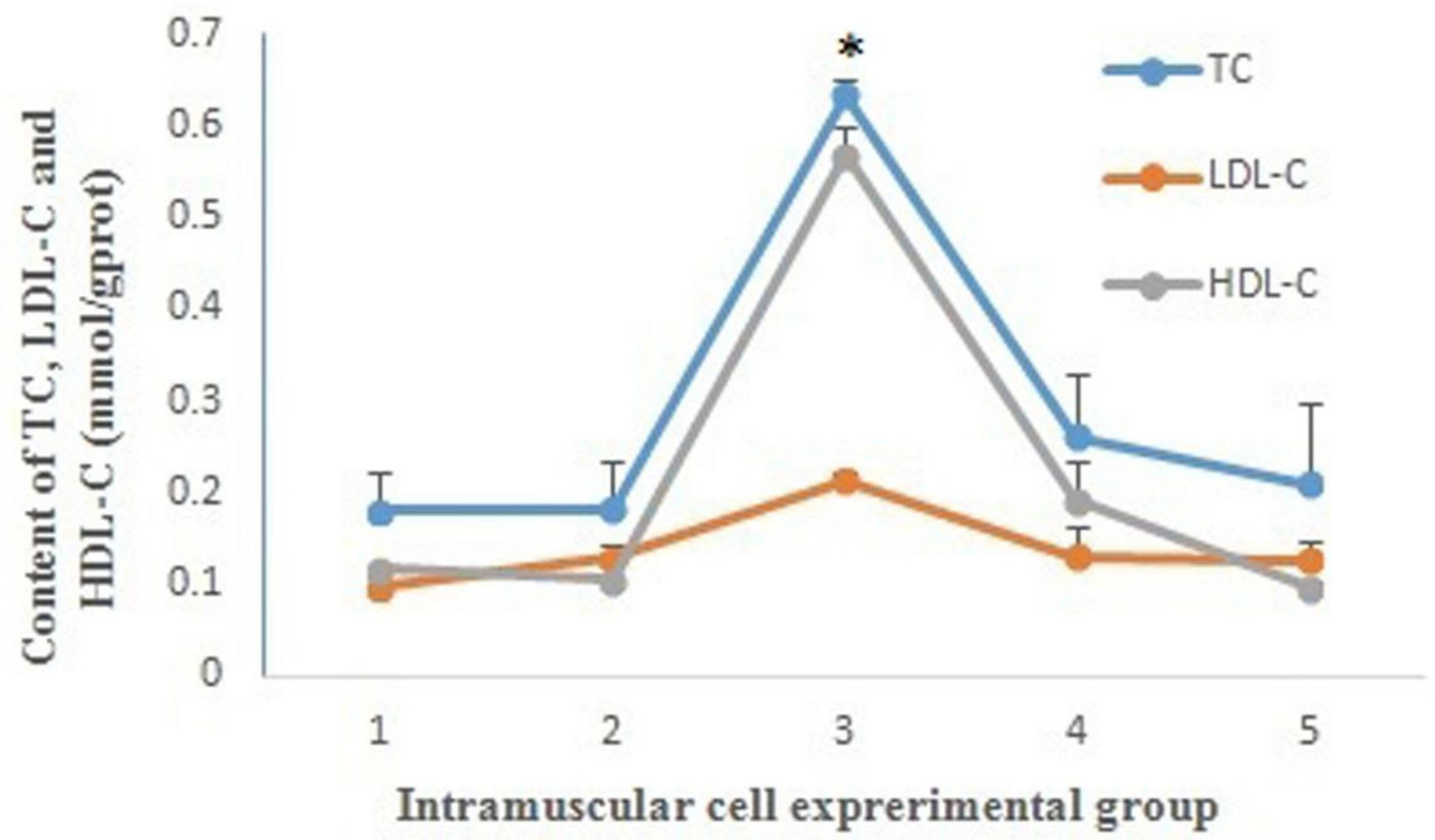

Figure 5

The levels of TC, LDL-C, and HDL-C in five different experimental groups. 1: adipogenic group (control group), 2: adipogenic + overexpression NC, 3: adipogenic + HMGR overexpression group, 4: adipogenic + interference NC, and 5: adipogenic + HMGR interference group. NC, negative control. 


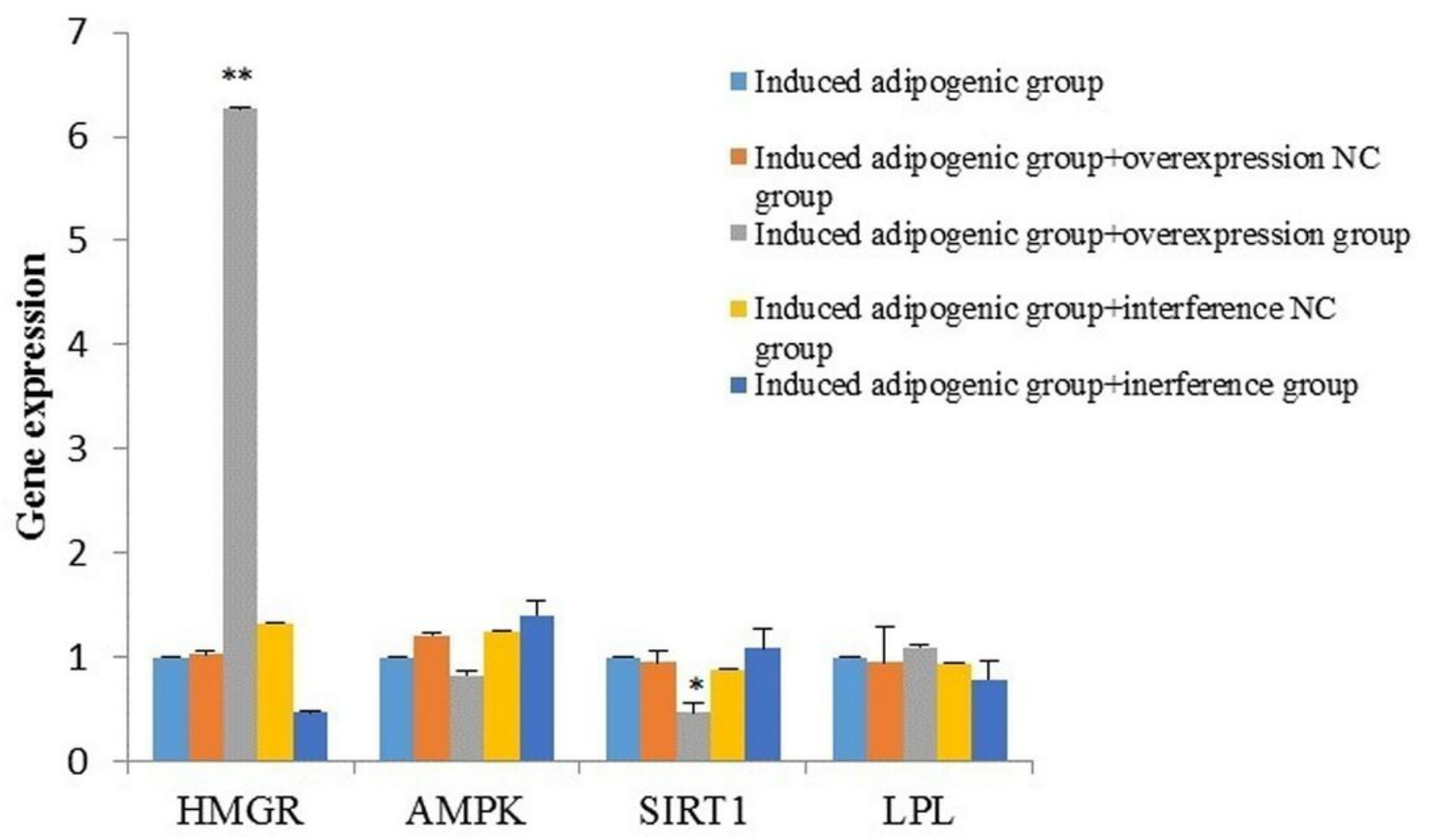

Figure 6

Gene expression of HMGR, AMPK, SIRT1, and LPL in intramuscular adipose cells.

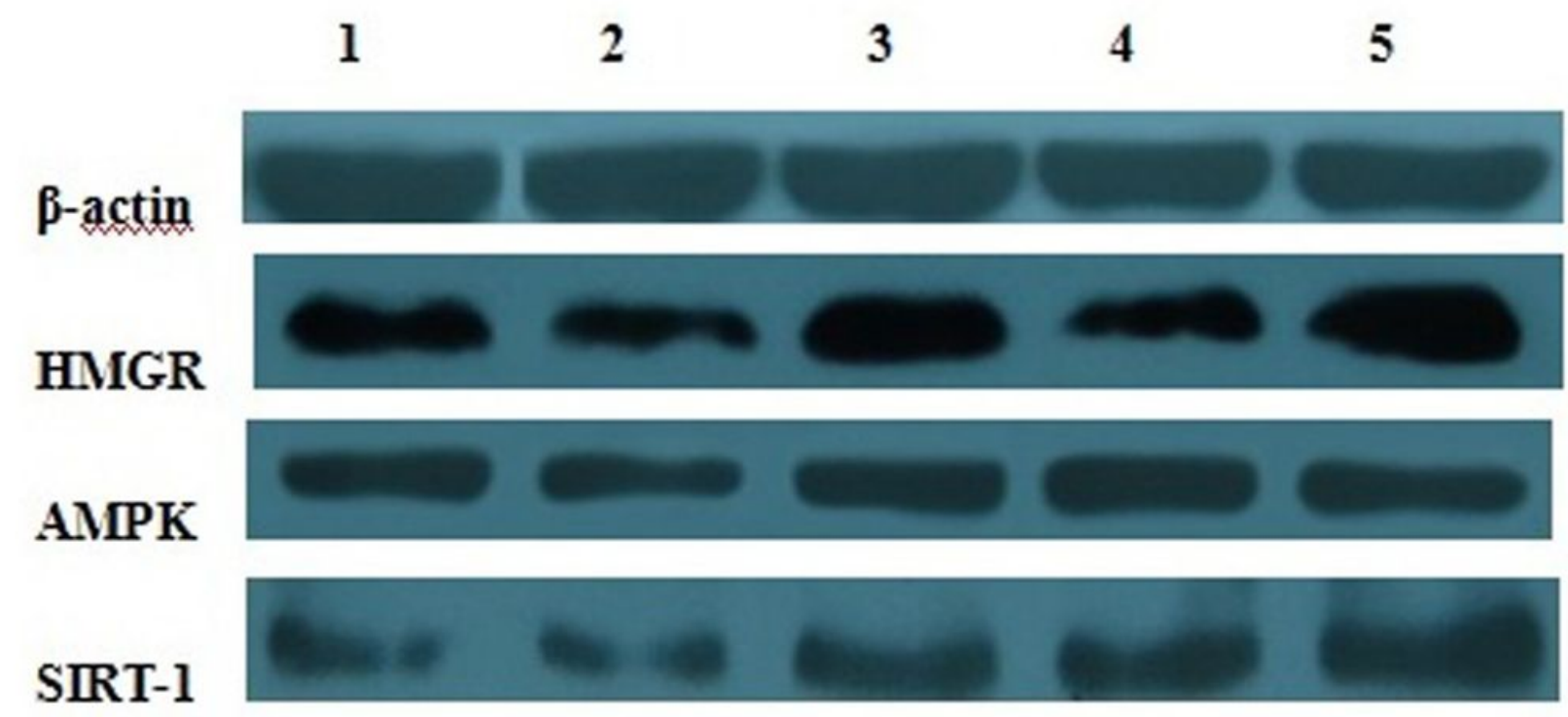

LPL

Figure 7 
Western blotting of HMGR, AMPK, SIRT1, and LPL in the five experimental groups. Column 1: adipogenic group (control group), column 2: adipogenic group + overexpression NC, column 3: adipogenic + HMGR overexpression group, column 4: adipogenic + interference NC, and column 5: adipogenic + HMGR interference group. NC, negative control. 